\title{
Doenças Ocupacionais Respiratórias - Perspetivas Atuais
}

\author{
António Jorge Correia de Gouveia Ferreira ${ }^{1}$ \\ ${ }^{1}$ Faculdade de Medicina da Universidade de Coimbra, Portugal; Email: aferreira@fmed.uc.pt.
}

\begin{abstract}
Resumo
As doenças ocupacionais são uma das causas mais significativas de morte e incapacidade relacionada com o trabalho. Dentro das múltiplas patologias desta família que podem afetar o ser humano, tomam principal destaque as afeções ocupacionais de etiologia respiratória, quer pelo seu significado a nível nacional e internacional, quer sobretudo pela sua gravidade e anos potenciais de vida perdidos. Constituem uma causa significativa de sintomatologia respiratória no trabalhador, bem como de absentismo, incapacidade e morte. Dentro da grande variedade de doenças ocupacionais respiratórias, o autor dá particular destaque às Pneumoconioses (provocadas pela inalação de poeiras minerais, quer sejam fibras ou partículas e sua subsequente resposta pulmonar, com particular impacto para a inalação de sílica cristalina respirável e amianto); Pneumonite de Hipersensibilidade Profissional (doença pulmonar complexa causada por uma reação imunitária desencadeada após a exposição respiratória a uma ampla variedade de antigénios; Asma Ocupacional (caracterizada por limitação variável do fluxo aéreo e/ou hiper-reatividade das vias aéreas, devidas a causas e condições atribuíveis a um ambiente de trabalho em particular e não a estímulos encontrados fora do local de trabalho) e Neoplasias Respiratórias Ocupacionais, com particular destaque para o cancro do pulmão e o mesotelioma pleural maligno).
\end{abstract}

Palavras-chave: asbestose; asma; cancro ocupacional; doenças ocupacionais; pneumoconioses; silicose.

\begin{abstract}
Occupational diseases are one of the most significant causes of death and disability related to work. Within the multiple occupational diseases that can affect the human being, the respiratory diseases related to the occupation are one of the most important, due to their national and international significance, involving high clinical severity and potential years of life lost. They constitute a significant cause of respiratory symptomatology in the worker, as well as absenteeism, disability and death. Within the wide range of occupational respiratory diseases, the author emphasizes the Pneumoconiosis (caused by the inhalation of mineral dust, whether fibers or particles and the subsequent lung response, with a particular impact on the inhalation of crystalline silica and asbestos); Occupational Asthma (characterized by variable airflow limitation and / or airway hyper responsiveness, due to causes and conditions of the respiratory tract attributable to a particular work environment) and occupational respiratory Neoplasms, with particular emphasis on lung cancer and malignant pleural mesothelioma).
\end{abstract}

Keywords: asbestosis; asthma; occupational cancer; occupational diseases; pneumoconiosis; silicosis.

\section{Introdução}

O Sistema Respiratório constitui a principal interface entre o ser humano e o meio ambiente. Naturalmente, qualquer exposição inalatória significativa, com múltiplas particularidades distintas no que diz respeito ao tipo de substâncias inaladas, à sua concentração, às suas dimensões, aos seus aspetos físico-químicos e à sua interação com a biologia humana, é passível de provocar danos a curto ou longo prazo.

Particular atenção tem vindo a ser dada às exposições ambientais e aos efeitos da poluição atmosférica sobre a Saúde, de tal forma que muitas doenças (respiratórias e não só) parecem apresentar uma fração epidemiológica de risco que pode ser atribuída ao ambiente interior ou exterior. 
As agressões inalatórias diárias sofridas a nível individual, bem como os mecanismos potenciais de exposição ambiental sofrida in utero, podem resultar no aumento da incidência e prevalência de várias patologias, onde se destaca a asma brônquica, a doença pulmonar obstrutiva crónica (DPOC), vários tipos de neoplasias (nomeadamente alguns cancros de pulmão e do sistema hematopoiético) e algumas doenças cardiovasculares, apenas para citar alguns dos exemplos mais reconhecidos.

A exposição a inúmeros agentes ambientais e ocupacionais tem um impacto significativo na saúde humana e a própria forma como surgem todos os dias riscos emergentes, novas tecnologias de trabalho e novas substâncias poluentes leva a que, no futuro, uma parcela cada vez mais significativa das doenças respiratórias (entre outras) seja atribuída a estas situações (Huang, Ghio, \& Maier, 2012).

\section{Principais Patologias Respiratórias de Etiologia Profissional Pneumoconioses}

As pneumoconioses são um grupo de doenças ocupacionais provocadas pela inalação de poeiras minerais (quer sejam fibras ou partículas) e pela subsequente resposta pulmonar a esta inalação (Banks \& Parker, 1998; Ghio, 2012).

Milhões de trabalhadores continuam a executar tarefas diárias com elevado risco para o desenvolvimento de pneumoconioses, especialmente silicose, asbestose e pneumoconiose do trabalhador do carvão (PTC - Coal Workers' Pneumoconiosis - CWP). A associação com outras doenças, nomeadamente, neoplasias da pleura e do pulmão, tuberculose e doença pulmonar obstrutiva crónica (DPOC), causam habitualmente um elevado nível de incapacidade.

Trata-se de um problema com grande expressão mundial atual: por exemplo, na China, as pneumoconioses correspondem a cerca de $80 \%$ de todas as doenças ocupacionais respiratórias, enquanto no Vietname, cerca de $75,7 \%$ do total de casos de doença ocupacional com compensação pertence a este grupo nosológico. No Brasil, 6,6 milhões de trabalhadores encontram-se provavelmente expostos a poeiras contendo sílica cristalina respirável (ILO 2013).

A Organização Mundial da Saúde (OMS) estima que pelo menos 1,7 milhões de trabalhadores nos Estados Unidos estão expostos à sílica e $10 \%$ estão em risco de desenvolver silicose (Ahasic \& Christiani, 2011).

Estudos epidemiológicos em países em desenvolvimento mostraram que entre $30 \%$ a $50 \%$ dos trabalhadores de indústrias primárias e de sectores de alto risco podem sofrer de silicose ou outras pneumoconioses (ILO, 2013).

Há três critérios major para o diagnóstico de uma pneumoconiose: uma exposição suficiente a uma poeira mineral indutora da doença, durante um período de latência apropriado; o reconhecimento de um padrão radiológico que vai ao encontro do estabelecido em termos internacionais e a comprovação da ausência de uma patologia que possa mimetizar uma pneumoconiose, nomeadamente a fibrose pulmonar idiopática, as micobacterioses e as doenças intersticiais pulmonares relacionadas com doenças do colagénio (Banks \& Parker, 1998).

As principais pneumoconioses têm um elevado impacto na saúde dos trabalhadores, pois vão desencadear uma resposta fibrogénica pulmonar, enquanto um outro grupo menos frequente de pneumoconioses se pode considerar como hipo ou afibrogénica e se relaciona essencialmente com a deposição de partículas inorgânicas inaladas, sem a subsequente resposta fibrogénica progressiva. A este nível, citam-se a estanose, a siderose, a antracose e a baritose, entre outras (Cordeiro, 1995).

Uma abordagem possível na avaliação de um doente com pneumoconiose passa por incluir uma história clínica (com ênfase na história de doença respiratória, história ocupacional e tabágica), exame físico, testes de função pulmonar (espirometria, volumes pulmonares e capacidade de difusão) e radiografia de tórax (Ghio, 2012).

A tomografia computorizada do tórax, nomeadamente com algoritmo de alta resolução (TCAR), é muito útil na avaliação do doente com pneumoconiose, devido à sua maior sensibilidade e capacidade de caracterização pulmonar, bem como devido a situações em que se exige um diagnóstico diferencial mais complexo. A TCAR é particularmente útil na criação de uma categorização precisa das alterações do parênquima em cada tipo de pneumoconiose (Akira, 2002; Savranlar et al., 2004; Begin et al., 1991).

Apesar das pneumoconioses poderem ser definidas em termos histopatológicos, raro é que seja necessária a obtenção de uma biópsia, (Ghio, 2012) exceto quando o diagnóstico diferencial se revela difícil.

$\mathrm{Na}$ ausência de exame patológico do tecido pulmonar, os critérios habitualmente aceites recomendam que o diagnóstico de pneumoconiose seja estabelecido após uma análise cuidadosa de todos os achados clínicos relevantes, bem como a existência de uma história fidedigna de exposição e um intervalo de tempo adequado entre esta e a deteção das alterações (Ghio, 2012, 1986). 
O Comité ad hoc da Assembleia Científica em Saúde Ambiental e Ocupacional da American Thoracic Society (ATS), considerou os seguintes achados clínicos como sendo de valor reconhecido para a asbestose, mas também extensíveis à outras pneumoconioses: evidência radiográfica torácica de pequenas opacificações, um padrão restritivo de compromisso pulmonar com capacidade vital forçada abaixo do limite inferior do normal, uma capacidade de difusão abaixo do limite inferior do normal e crepitações auscultatórias pulmonares não modificáveis através de tosse (Ghio, 2012).

A patogénese das pneumoconioses baseia-se essencialmente numa resposta alveolar, com produção de citocinas inflamatórias, espécies reativas de oxigénio (ROS) e enzimas proteolíticas, levando à subsequente alteração pulmonar (Fujimura, 2000).

Nas pneumoconioses fibrogénicas a resposta patogénica é similar entre si: após a fagocitose das partículas inaladas, os macrófagos são ativados e libertam vários mediadores inflamatórios, causando uma resposta inflamatória com lesão celular e disrupção da matriz extracelular. Segue-se uma fase de reparação, em que fatores de crescimento estimulam o recrutamento e proliferação de células mesenquimatosas, provocando neovascularização e re-epitelização das áreas mais afetadas; durante esta fase, os mecanismos de reparação observados são anormais ou não controlados e levam ao desenvolvimento de fibrose concomitante. Adicionalmente, o recrutamento neutrofílico observado pode contribuir para uma amplificação da resposta alveolar ao secretar fator de necrose tumoral alfa (TNF $\alpha$ ) ou interleucina-1. Vários estudos demonstram que os macrófago salveolares de indivíduos com silicose ou pneumoconiose do trabalhador do carvão, libertam espontaneamente quantidades significativas de fatores de crescimento tais como o PDGF (fator de crescimento derivado das plaquetas), IGF (fator de crescimento insulin-like) e FGF (fator de crescimento dos fibroblastos) (Brichet et al., 1999).

Vários fatores podem influenciar o risco de desenvolvimento de uma pneumoconiose: suscetibilidade individual, natureza e duração da exposição, dimensão, morfologia da superfície e caraterísticas físico-químicas das partículas inaladas, entre outras (Brichet et al., 1999).

\section{Silicose}

A silicose é uma Pneumoconiose que resulta da inalação de poeiras contendo sílica sob a forma de dióxido de sílica (SiO2) em fração respirável, sendo que a forma mais frequente de sílica cristalina é o quartzo, seguida pela cristobalite e tridimite (Brichet et al., 1999; Leung, Yu, \& Chen, 2012). A sílica amorfa, presente por exemplo nas terras diatomáceas, só constitui risco se calcinada por exposição a temperaturas elevadas (Greenberg, Waksman, \& Curtis, 2007).

Diversos tipos de exposição ocupacional podem desencadear silicose. Destacam-se trabalhos na indústria extrativa, fundições de ferro, aço ou outros metais onde se utilizam moldes de areia, cerâmicas, produção de tijolos refratários, fabricação de vidros (tanto na preparação como também no uso de jato de areia usado para sua opacificação), perfuração de rochas na indústria da construção, decapagem com jato de areia, execução de trabalho em mármore, ardósia, granito e outras pedras decorativas, fabricação de material abrasivo, fabricação de cimento, escavação de poços, construção civil, tratamento de jeans por jato de areia e trabalho com próteses dentárias (Leung, Yu, \& Chen, 2012).

O granito, uma das rochas mais abundantes em Portugal, contém 25-40\% de sílica (Greenberg, Waksman, \& Curtis, 2007).

A apresentação clínica e a evolução da silicose estão muito dependentes da interação entre o hospedeiro e o ambiente ocupacional. Assim, a silicose pode apresentar-se sob diversas formas: silicose crónica (simples ou complicada), silicose acelerada e silicoproteinose (Santos et al. 2010; Greenberg, Waksman, \& Curtis, 2007, Leung, Yu, \& Chen, 2012).

A silicose aguda ou silicoproteinose desenvolve-se de forma aguda após exposições maciças a sílica cristalina e mimetiza a proteinose alveolar, com presença de exsudato intra-alveolar rico em lipoproteínas que coram positivamente pela coloração PAS (Coloração ácido periódico-Schiff).

A silicose acelerada é similar à silicose crónica, mas desenvolve-se em períodos de tempo mais curtos, habitualmente 5 a 10 anos após o início da exposição profissional. A silicose crónica é a forma mais habitualmente observada a nível ocupacional, começando a ser aparente após um período de cerca de 10 a 15 anos de indução.

A silicose crónica simples manifesta-se pela presença de pequenos nódulos silicóticos, de predomínio nos lobos superiores do pulmão e nos gânglios linfáticos e constituídos por diversas camadas de tecido conjuntivo, com partículas de sílica cristalina no seu interior.

A silicose crónica complicada resulta da coalescência dos nódulos silicóticos, formando massas sob a forma de conglomerados fibróticos que ocupam áreas cada vez mais significativas do pulmão (fibrose maciça progressiva) e podem cavitar centralmente. Outras condições que podem estar também associadas à exposição ocupacional a sílica cristalina são a tuberculose e outras 
micobacterioses, a artrite reumatóide, a esclerodermia, a síndroma de Sjögren e a doença renal crónica. Em 1997 e posteriormente em 2009, a Agência Internacional de Investigação sobre o Cancro (IARC) classificou a cristobalite e também as suas outras formas cristalinas, como carcinogénio do Grupo I, tal como o fez o Programa Nacional de Toxicologia dos EUA (Leung, Yu, \& Chen, 2012; Santos et al., 2010).

Habitualmente o diagnóstico é realizado pela conjugação de uma história clínica sugestiva com imagiologia torácica característica, nomeadamente através dos sistemas de classificação estandardizados sugeridos pela Organização Internacional do Trabalho (OIT) (ILO, 2011). Raramente é necessária a realização de biópsias pulmonares, que são habitualmente muito esclarecedoras, mas cujo papel só se torna fundamental no diagnóstico diferencial com outros quadros clínicos. O líquido de lavagem broncoalveolar (LBA) apresenta geralmente uma celularidade aumentada com um padrão misto linfocítico/granulocítico com uma linfocitose de predomínio T-CD8, com diminuição da relação CD4:CD8 (Cordeiro, 1995; Cordeiro et al.; 2007).

O estudo funcional respiratório revela de forma mais frequente uma síndroma restritiva, associada a uma diminuição da capacidade de difusão (Leung, Yu, \& Chen, 2012; Santos et al., 2010). A prevenção é fundamental, uma vez que não existem terapêuticas com sucesso significativo.

\section{Asbestose}

Amianto ou asbesto é um termo geral aplicado a um grupo heterogéneo de minerais silicatados e hidratados de magnésio e que têm em comum uma tendência para separar-se em fibras (Dodson \& Hammar, 2005).

As principais formas de amianto são crisótilo (amianto branco - forma serpentina); crocidolite (amianto azul); grunerite (amosite, amianto castanho); actinolite; antofilite e tremolite (2006).

Estas fibras, inaladas e transportadas para o interior do sistema respiratório, podem desenvolver diversos tipos de patologia, em que se destaca a formação de placas pleurais, o espessamento pleural difuso, a asbestose, que constitui uma Pneumoconiose com resposta fibrogénica pela inalação de amianto, o derrame pleural benigno induzido por amianto e várias formas de neoplasia (cancro do pulmão e mesotelioma pleural maligno).

O consumo máximo de amianto na Europa ocorreu um pouco antes de 1980, sendo usado em inúmeras situações, em que se destacam as suas propriedades ignífugas, de resistência à tração e isolantes térmicas e acústicas. A proibição total da utilização e comercialização de produtos que contenham amianto (pela aplicação da Diretiva 1999/77/CE da Comissão Europeia) deu-se apenas a 1 de janeiro de 2005 (2006).

Todas as variedades de amianto têm um potencial cancerígeno. O Valor Limite de Exposição (VLE) segundo o Decreto-Lei n.ㅇ 266/2007, de 24 de julho, é fixado em 0,1 fibras/cm3 para todos os tipos de fibras de amianto (UONIE/ACSS 2008).

O diagnóstico clínico das doenças relacionadas com o amianto é baseado na entrevista detalhada ao doente, dados ocupacionais de exposição ao amianto, período de latência considerado adequado, sinais e sintomas, achados radiológicos e exames complementares de diagnóstico compatíveis. A confirmação histopatológica é necessária na suspeita de doenças malignas relacionadas com o amianto e na eventualidade da existência de outros diagnósticos diferenciais (Tossavainen, 1997).

A asbestose é definida como uma fibrose pulmonar intersticial difusa que é consequência da exposição ao amianto. Nem os aspetos clínicos, nem as alterações pulmonares observadas são suficientemente diferentes das de outras causas de fibrose intersticial que permitam o diagnóstico seguro sem uma história de exposição significativa a poeiras de amianto no passado ou a deteção de fibras ou corpos de amianto no tecido pulmonar muito em excesso do que é usualmente visto na população em geral (Tossavainen, 1997).

A asbestose é geralmente provocada por níveis relativamente altos de exposição, associados a sinais radiológicos de fibrose do parênquima pulmonar. A sua clínica, inespecífica, engloba tosse seca, dispneia de esforço e fervores auscultatórios teleexpiratórios de predomínio basal. Na radiografia torácica é habitualmente aparente a presença de infiltrados reticulomicronodulares nas bases pulmonares (Pipavath, Godwin \& Kanne, 2010). A Tomografia Computorizada de Alta Resolução (TCAR) permite uma caracterização mais precoce da doença, observando-se de modo geral, espessamentos dos septos inter e intra-lobares na periferia do pulmão, estrias parenquimatosas estendendo-se entre a pleura e o pulmão, aspetos em "favo de mel", atelectasias discais e densidades parenquimatosas subpleurais (Begin, 1999). Em cerca de 10\% dos indivíduos com asbestose histologicamente comprovada, a radiografia torácica é normal. 
O diagnóstico patológico de asbestose é raramente obtido, mas exige a prova da presença de uma doença pulmonar intersticial com corpos de amianto em tecido humano (dois ou mais por $\mathrm{cm} 2$ por campo microscópico) (Ghio, 2012). A exposição ao amianto é geralmente referida em termos de dose total ou cumulativa. A dose final é resultante do tempo de exposição (em anos) e da intensidade da exposição, tal como definido pela concentração média de fibras por centímetro cúbico $(\mathrm{f} / \mathrm{cm} 3)$ de ar do local de trabalho. Somente as fibras superiores a $5 \mu \mathrm{m}$ de comprimento são contadas. Assim, um indivíduo exposto a duas fibras/cm3 durante dez anos, teria uma exposição total de 20 (Dodson \& Hammar, 2005). Os critérios do Relatório do Consenso de Helsínquia afirmam que a asbestose "pode ocorrer" com exposições na ordem de 25. Não há nenhuma evidência convincente de que a asbestose ocorra com exposições inferiores a dez (Tossavainen, 1997; Dodson \& Hammar, 2005).

O líquido de lavagem broncoalveolar (LBA) obtido por broncofibroscopia é uma ferramenta útil na asbestose, não só para avaliar a presença de uma alveolite linfocítica (e/ou neutrofílica) moderada, maior nas fases precoces e menor em doença mais avançada, e geralmente associada a relação CD4:CD8 aumentada, mas essencialmente para identificar e quantificar as fibras de amianto. Supõe-se que o número de corpos asbestósicos encontrados no LBA reflete a sua concentração no pulmão (Begin, 1999; Cordeiro et al., 2007). A recuperação de mais do que um corpo asbestósico/ml de LBA indica uma alta probabilidade de exposição ocupacional substancial ao amianto (1986).

Os corpos asbestósicos (ou ferruginosos) podem ser vistos ao microscópio em LBA, expetoração induzida e tecido pulmonar. Estes são corpos em forma de halter, com cerca de 20 a $150 \mu \mathrm{m}$ de comprimento, constituídos por fibras cobertas por uma camada de mucopolissacarídeos. O pigmento de ferro (que resulta da degradação da hemoglobina) fá-los parecer acastanhados (Ahasic \& Christiani 2011, 1986). O amianto anfíbolo forma a maioria dos corpos asbestósicos e é mais persistente no organismo do que o crisótilo (1986).

O estudo funcional respiratório revela habitualmente alterações similares às de outras pneumoconioses: síndroma restritiva e diminuição da capacidade de difusão.

Mais uma vez, a prevenção e legislação de âmbito ocupacional desempenham um importante papel, uma vez que não existe terapêutica específica para a doença (Dodson \& Hammar 2005).

\section{Pneumonite de Hipersensibilidade de Etiologia Ocupacional}

A pneumonite de hipersensibilidade ( $\mathrm{PH})$, também apelidada previamente por alguns autores de alveolite alérgica extrínseca (AAE), é uma doença pulmonar complexa causada por uma reação imunitária desencadeada após a exposição respiratória a uma ampla variedade de antigénios, incluindo microrganismos, proteínas de origem vegetal e animal e compostos químicos. Cerca de 300 agentes já foram identificados como potencialmente causadores da doença (Ho \& Kuschner, 2012; Hirschmann, Pipavath, \& Godwin, 2009; Agostini et al., 2004; Fink et al., 2005).

Ao longo dos últimos 10 a 15 anos tem havido um crescente interesse nesta patologia, tendo inclusive sido criado um grupo de estudo da PH, e publicado um importante documento de trabalho revelando a cooperação entre o National Heart, Lung and Blood Institute dos EUA e o Departamento de Doenças Raras do National Institutes of Health dos EUA (Fink et al., 2005; Lacasse, Girard, \& Cormier, 2012).

Este grupo definiu a PH como uma doença pulmonar que manifesta sintomas de dispneia e tosse e que é resultante da inalação de um antigénio ao qual o doente tinha sido previamente sensibilizado. Algumas considerações se colocam: (1) A PH é uma doença pulmonar com ou sem manifestações sistémicas (tais como a febre e a perda de peso), (2) é causada pela inalação de um antigénio ao qual o indivíduo (por exemplo, o trabalhador) está sensibilizado, (3) a sensibilização e exposição isoladas na ausência de sintomas não definem a doença, uma vez que muitos indivíduos expostos desenvolvem uma resposta imune que se manifesta pela presença de anticorpos IgG para o antigénio em causa, muitas vezes com a presença de grande número de linfócitos nos seus pulmões, mas nunca vêm a desenvolver doença clínica (Lacasse, Girard, \& Cormier, 2012).

Pensa-se que na Europa, as PH representem cerca de 4 a 15\% de todas as doenças intersticiais pulmonares, e destas um número significativo pode ser correlacionado com o trabalho (Thomeer et al., 2001; Lacasse, Girard, \& Cormier, 2012). Por exemplo, durante a sua vida, um agricultor tem uma probabilidade entre 0,5 a 3\% de desenvolver PH (Bang et al., 2006).

Quadro 1. Causas ocupacionais de Pneumonite de Hipersensibilidade 


\begin{tabular}{|c|c|c|}
\hline Agente & Fonte & Doença \\
\hline \multicolumn{3}{|l|}{ Agentes microblanos } \\
\hline \multirow{2}{*}{ Actinomicerss termofilicos } & Feno húmido & Pul mào do fazendeiro \\
\hline & Agua quentecontaminada & Pul mào do humidificador \\
\hline Thermos ctino myces sacchari & Cana dea çucar & Bagassose \\
\hline Thermoo ctino myces vulgaris & Cogumei os en decompos içào & Pul mào do trabal hador de cogumel os \\
\hline Aspergillus dovotus & Malte húmido & Pul mão do trabal hador do malte \\
\hline Aspergillus sp. & Fu ngos do tabaco & Pul mào do trabal hador do tabaco \\
\hline Penicillium cosei & Quejo bo lorento & Pul mào do lavador de quejo \\
\hline Aureo bosidium pullulans & Poeira des equoia & Sequoi ose \\
\hline Rhyzo pus $\$ p$-; Mucor $s p$. & Aparas de madeira & Pul mào do se rrador \\
\hline Penicillum sp. & Cortiça bolorenta & Suberose \\
\hline Enzimas do Bacillus subtilis & Detergentes & Pul mào do trabal hador dos deter gentes \\
\hline Altemario sp. & Pasta de pape contaminada & Pul mào do trabal hador da pasta de pape \\
\hline \multicolumn{3}{|l|}{ Proteinas } \\
\hline Proténas de origemaviánia & várias aves & Pul mào do criador de aves \\
\hline Proténas de pelos animais & Pel os animais & $\begin{array}{l}\text { Pul mào do pele éro; pul mào do trabal hador de } \\
\text { laboratório de experimentaçào animal }\end{array}$ \\
\hline Proténas da concha de mol us cos & Pó das conchas de mol us cos & Pul mào do trabal hador de mol us cos \\
\hline \multicolumn{3}{|c|}{ Quimicos de balxo peso molecular } \\
\hline Is ocianatos & Piásticos, tintas & Pul mào do pintor \\
\hline Anidridos & Tintas & Pul mào do trabal hador de plás fcos \\
\hline Qu'micos epoxy & Resinas epoxy aquecidas & Pul mào das resinas epoxy \\
\hline
\end{tabular}

Adaptado de Ho, (2012) e de Salvaggio, (1998) (Ho \& Kuschner, 2012; Salvaggio, Vockroth, \& Lopez, 1998).

Historicamente, investigadores portugueses têm desenvolvido valiosos trabalhos nesta área, nomeadamente na suberose e na doença pulmonar do criador de aves (Winck et al., 2004; Morais et al., 2004). As principais causas de PH podem ser divididas em três grandes categorias (agentes microbianos, proteínas animais e químicos com baixo peso molecular) (Ho \& Kuschner, 2012). No Quadro 1, apresentam-se alguns dos agentes etiológicos implicados.

A clínica da doença pode assumir formas mais agudas, com febre e mal-estar geral e por vezes uma síndroma de tipo gripal (flulike), formas subagudas e crónicas, com dispneia de esforço, baqueteamento digital, fervores e ralas à auscultação pulmonar. Contudo, Lacasse e col. referem, após estudo de coortes significativas de doentes, que existem sobretudo dois subgrupos (doença aguda e doença crónica), sendo difícil estabelecer critérios precisos para as formas subagudas (Lacasse, Girard, \& Cormier, 2012). Os mesmos autores preconizaram seis preditores significativos para a presença de PH (exposição a um agente etiológico conhecido, precipitinas positivas, recorrência sintomatológica, crepitações inspiratórias à auscultação, sintomatologia após 4-8 horas do início da exposição e perda de peso). A presença dos seis preditores identificou corretamente $98 \%$ de toda a coorte estudada, enquanto nenhum doente apresentou ausência de todos os preditores.

No estudo da PH e após uma cuidadosa história clínica ocupacional e ambiental, destacam-se as seguintes características (Akira, 2002; Ho \& Kuschner, 2012; Salvaggio, Vockroth, \& Lopez, 1998; Lacasse, Girard, \& Cormier, 2012, Costabel, Bonella, \& Guzman, 2012; Zacharisen \& Fink, 2011; Thaon et al., 2006):

- Radiografia torácica habitualmente normal nas fases mais precoces, ou então com infiltrados bilaterais simulando por vezes pneumonia nas formas agudas. Podem estar presentes alterações do tipo reticulo-micronodular.

- TCAR com nodulação centrilobular, áreas de hiperatenuação em "vidro despolido" de predomínio não inferior, com possível padrão em mosaico com áreas de encarceramento aéreo; em fases avançadas com reticulação, bronquiectasias de tração e áreas em "favo", traduzindo fibrose.

- Estudo da função respiratória com padrão restritivo e diminuição da capacidade de difusão, com a exceção do pulmão do fazendeiro, que pode assumir um padrão obstrutivo preferencial.

- Presença de anticorpos IgG positivos para os antigénios causadores da doença (previamente conhecidos como precipitinas, por serem determinados através de técnicas de imunoprecipitação). Estes não são patognomónicos, uma vez que muitos trabalhadores expostos apresentam positividade para estes anticorpos, sem doença aparente. 
- Possibilidade da presença de positividade em testes de provocação com o agente específico (embora não haja critérios estandardizados, ao contrário do que acontece na asma ocupacional).

- LBA revelando habitualmente uma linfocitose > 30\%. A inexistência de linfocitose no LBA exclui, em princípio, a doença ativa. A relação CD4:CD8 é habitualmente baixa, mas já foram observadas situações em que sucede o contrário, apresentando valores próximos dos presentes na sarcoidose (Cormier et al., 1986; Lacasse, Girard, \& Cormier, 2012).

A terapêutica da PH está intimamente ligada à rápida eviç̧ão do estímulo etiológico ocupacional, desempenhando aqui um papel preponderante as atividades de Saúde e Segurança no Trabalho, nomeadamente no que toca à prevenção primária e secundária. Em termos farmacológicos, é essencialmente utilizada a corticoterapia sistémica oral, em formas agudas graves ou em formas crónicas com repercussão funcional ou rapidamente progressivas, com resultados diversos consoante as séries clínicas referenciadas. Poderão igualmente ser usados fármacos imunossupressores, tais como a azatioprina (Costabel, Bonella, \& Guzman, 2012; Lacasse, Girard, \& Cormier, 2012; Salvaggio, Vockroth, \& Lopez, 1998).

\section{Asma Ocupacional}

A asma ocupacional é uma doença caracterizada por limitação variável do fluxo aéreo e/ou hiper-reatividade das vias aéreas, devidas a causas e condições atribuíveis a um ambiente de trabalho em particular e não a estímulos encontrados fora do local de trabalho. A asma ocupacional é a doença pulmonar respiratória mais comum em países desenvolvidos (Mapp \& Boschetto, 2003; Vandenplas \& Malo, 2003; Baur et al., 2012; Tarlo et al., 2008; Baur \& Sigsgaard 2012; Henneberger et al., 2011; Nicholson, Cullinan, \& Burge, 2012).

É sabido que um largo espectro de exposições ocupacionais pode induzir um agravamento de uma asma brônquica préexistente, nomeadamente a poeiras, aeroalergéneos do ambiente indoor e outdoor e detergentes e soluções de limpeza (Szema, 2012).

Mais de 250 agentes foram associados etiologicamente à asma ocupacional. Os mais frequentes são os di-isocianatos, farinha e pó de diversos grãos, partículas em suspensão de alimentos (principalmente peixes), resinas, látex, partículas animais (especialmente de animais de laboratório), aldeídos e pós de madeira (Ribeiro \& Tarlo, 2012; Tan \& Bernstein, 2014).

A prevalência da asma relacionada com o trabalho não é conhecida, tendo-se estimado que cerca de 10 a $15 \%$ de toda a asma brônquica do adulto possa ser atribuída ao local de trabalho, incluindo-se nestes números os casos de asma ocupacional e de asma exacerbada pelo local de trabalho (Blanc \& Toren, 1999). Outros autores referem valores na ordem dos $5-25 \%$ do total de casos (Baur et al., 2012). Os custos europeus totais atribuíveis à asma relacionada com o trabalho não são conhecidos em detalhe, mas estima-se que possam atingir 1,2 mil milhões de euros/ano (Baur \& Sigsgaard 2012).

Especificamente, várias definições têm sido propostas ao longo dos anos por diversos autores, havendo precisamente alguma área de indefinição no que toca à distinção entre asma ocupacional provocada por estímulos existentes apenas nos locais de trabalho e/ou também fora deles. Assim, de forma mais globalmente aceite, o termo asma ocupacional refere-se especificamente a asma provocada pelas exposições observadas no local de trabalho (Lemiere et al., 2013).

Algumas definições mais abrangentes e por isso mais inespecíficas, referem um termo geral de asma relacionada com o trabalho (work-related asthma), que inclui a asma ocupacional e a asma exacerbada pelo trabalho (work-exacerbated asthma) (Lemiere et al., 2013; Vandenplas \& Malo, 2003).

Malo e Vandenplas sugeriram em 2011 um esquema que considera algumas outras síndromas clínicas observáveis em ambiente laboral, como se pode ver na Figura 1. 


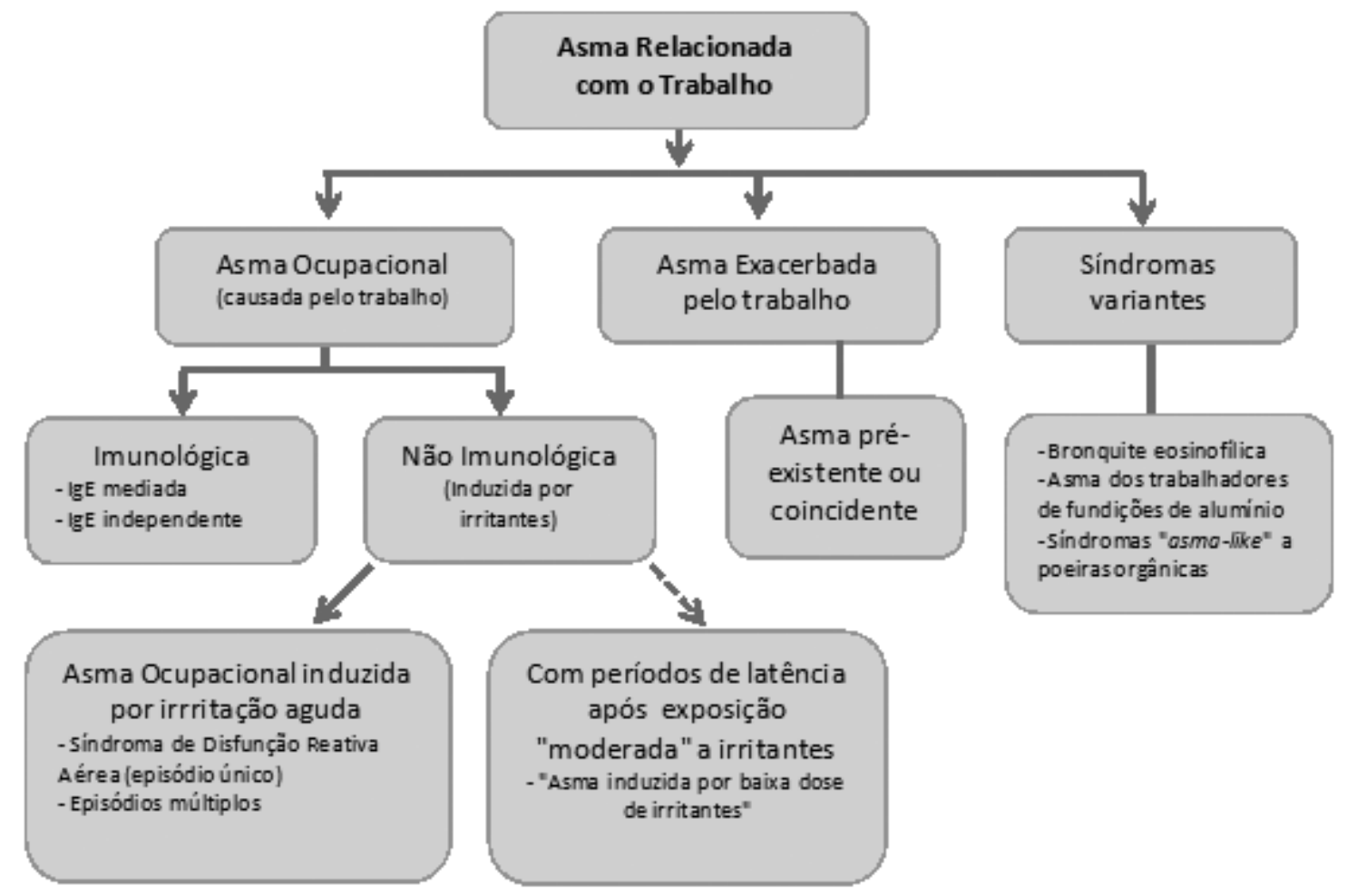

Figura 1. Esquema sugerido para definição de asma relacionada com o trabalho. [Adaptado de Malo e Vandenplas, 2011 (Malo \& Vandenplas, 2011)].

Dois tipos de asma ocupacional podem ser distinguidos, dependendo se são ou não mediados por mecanismos imunológicos: asma ocupacional imunológica e asma ocupacional não imunológica. Na primeira, observa-se habitualmente um período de latência durante o qual se processa a exposição e sensibilização ao agente ocupacional responsável. Dentro desta categoria encontra-se a asma ocupacional causada por todos os compostos de alto peso molecular e ainda alguns de baixo peso molecular (anidridos ácidos, sais de platina, alguns corantes). Nestas situações, estabeleceu-se um mecanismo imunológico IgE dependente inequívoco; de igual modo se encontra nesta categoria a asma ocupacional provocada por compostos de baixo peso molecular, através de mecanismos imunológicos não completamente conhecidos (di-isocianatos, cedro vermelho ocidental, acrilatos). Nas Guidelines do American College of Chest Physicians, esta última forma é conhecida como asma ocupacional induzida por sensibilizadores ("sensitizer-induced") (Tarlo et al. 2008; Bernstein et al., 2006; Vandenplas \& Malo, 2003).

A nomenclatura proposta pela Academia Europeia de Alergia e Imunologia Clínica sugere que a asma ocupacional mediada por mecanismos imunológicos (qualquer que seja a sua natureza exata) e que resulta em hipersensibilidade alérgica clínica, seja conhecida como asma ocupacional alérgica (Johansson et al., 2001).

A asma ocupacional não imunológica é essencialmente caracterizada pela ausência de um período de latência, que seria necessário para a habitual resposta imunológica após uma sensibilização do hospedeiro. Com efeito, a sintomatologia pode ocorrer poucas horas após uma única exposição irritante no local de trabalho; a entidade clínica mais descrita neste tipo de asma ocupacional é a síndroma de disfunção reativa das vias aéreas, que cursa com início das queixas (tosse, dispneia, pieira) nas primeiras 24 horas após a exposição, as quais não aliviam significativamente fora do local de trabalho, não havendo qualquer período de latência prévio e sendo comprovada a presença de um padrão obstrutivo e/ou hiper-reatividade brônquica inespecífica (Bernstein et al., 2006; Mapp \& Boschetto, 2003; Vandenplas \& Malo, 2003).

A asma exacerbada pelo trabalho descreve um agravamento de uma asma pré-existente, manifestando-se habitualmente por aumento da frequência ou severidade dos sintomas e/ou do consumo de fármacos para o seu tratamento.

O diagnóstico da asma ocupacional é um processo complexo, havendo necessidade de estabelecer nexos de causalidade inequívocos entre uma determinada exposição profissional e o advento das queixas, excluindo outras causas não observáveis a nível laboral. O diagnóstico da asma ocupacional deve ser investigado antes de aconselhar o trabalhador a abandonar o seu local de trabalho, já que a evicção da exposição prolongada pode influenciar a fiabilidade dos procedimentos de diagnóstico (Mapp \& Boschetto, 2003). As implicações médico-legais a este nível são muito importantes. 
Assim, o diagnóstico resulta essencialmente da integração de dados relativos à história clínica e ocupacional do doente, testes cutâneos de alergia, sempre que possível, pesquisa de IgE específica (nos casos de asma ocupacional imunológica IgE mediada), medições seriadas da função ventilatória no local de trabalho e fora deste, estudo funcional respiratório com provocação inespecífica e por vezes, se necessário, com o agente laboral suspeito.

\section{Doença Pulmonar Obstrutiva Crónica (DPOC) de Etiologia Ocupacional}

A DPOC é uma doença frequente, evitável e tratável e é caracterizada por uma obstrução persistente das vias aéreas, que é geralmente progressiva e está associada a uma resposta inflamatória crónica aumentada na sequência da inalação de partículas ou gases nocivos onde se podem encontrar seguramente diversos estímulos ocupacionais, não obstante a etiologia principal ser o fumo do tabaco. As exacerbações e co-morbilidades contribuem para a gravidade global da doença. A obstrução persistente resulta de uma combinação de doença difusa das pequenas vias aéreas e destruição do parênquima pulmonar (GOLD, 2014).

O termo "Bronquite Profissional" ou "Bronquite Industrial" muito usado sobretudo no Século XX e fazendo ainda parte, historicamente do léxico pneumológico, refere-se essencialmente à presença de queixas crónicas de tosse, dispneia e expetoração abundante em trabalhadores expostos a riscos inalatórios e é um pouco mais redutora do que a denominação "DPOC de etiologia profissional" pois esta última já envolve outras características, não apenas clínicas, no seu diagnóstico e supõe, também, a presença de limitação ventilatória obstrutiva demonstrável e fenómenos de destruição parenquimatosa pulmonar tipicamente associados, nomeadamente algumas formas de enfisema (Sunyer et al., 2005; Zock et al., 2001; Korn et al., 1987; Bakke et al., 1991).

A DPOC é uma síndroma com muitos fenótipos e com uma projeção mundial em constante crescimento. Por exemplo, na Europa estima-se uma prevalência de cerca de 5 a 10\% (consoante os critérios usados para o seu diagnóstico) e uma mortalidade de 18/100000 habitantes. Recentemente, o Programa BOLD (Burden of Obstructive Lung Diseases) documentou, em estudos espirométricos realizados a nível internacional, prevalência substancial da doença em nunca fumadores (3-11\%), o que está provavelmente relacionado com as exposições profissionais e/ou ambientais, bem como com o envelhecimento da população (Buist et al., 2007). Em Portugal, num estudo efetuado na região de Lisboa, estimou-se uma prevalência de DPOC de 14,2\%, em adultos com mais de 40 anos de idade (Barbara et al., 2013).

O Global Burden of Disease Study da OMS projetou que a DPOC, que ficou em sexto lugar como causa de morte em 1990, se vai tornar a terceira maior causa de morte no mundo em 2020; uma projeção mais recente estima que a DPOC será a quarta principal causa de morte em 2030 (Mathers \& Loncar, 2006; GOLD, 2014; Lopez et al., 2006).

Tal como no cancro de pulmão ocupacional, a DPOC ocupacional é em tudo sobreponível à DPOC de outras etiologias, nomeadamente a tabágica, o que coloca especial dificuldade na estratificação do risco atribuível entre vários fatores causais sinérgicos e concomitantes, o que pode, por exemplo, dificultar a atribuição médico-legal de incapacidade (Burge, 1999).

Entre as principais dificuldades no estudo da DPOC ocupacional referem-se:

- Os trabalhadores doentes são em tudo indistinguíveis dos casos de DPOC não ocupacional;

- Em geral, no início da prática laboral, observa-se em média, um melhor estado de saúde ("efeito do trabalhador saudável") com valores espirométricos que podem até ser mais altos do que o expectável;

- Os estudos prospetivos podem mostrar um abandono mais precoce pelos trabalhadores que desenvolvem maior agravamento funcional, a que corresponde mudança de trabalho ou aposentação precoces (Burge, 1999).

Nos agentes etiológicos ocupacionais mais frequentemente observados, destacam-se:

- Poeiras orgânicas (trabalho em corte e processamento de madeiras, exposição a poeiras de cereais, trabalho agrícola e na pecuária, trabalho em silos, havendo frequentemente contaminação microbiológica concomitante por endotoxinas e micotoxinas);

- Fumos e poeiras metálicas (alumínio, ósmio, cádmio, vanádio, usados sobretudo no trabalho metalúrgico, na indústria do aço, produção de zinco e soldadura de diversos tipos);

- Fumos da combustão diesel;

- Poeiras mistas, contendo minerais e material orgânico (indústria extrativa e construção) (Diaz-Guzman, Aryal, \& Mannino, 2012). 
Por outro lado, o fumo de tabaco ambiental pode, também, ser considerado uma causa de DPOC em nunca fumadores, nomeadamente em países sem legislação mais restritiva (Salvi, 2014).

\section{Cancro do Pulmão de Etiologia Ocupacional}

Historicamente, o nexo de causalidade entre exposições ocupacionais e cancro de pulmão começou a ser estabelecido no Século XIX, com a exposição a radão (Yu, Tse, \& Qiu, 2012). Desde aí, inúmeras outras exposições têm sido relacionadas com este tipo de neoplasia, que é uma das mais frequentes em termos ocupacionais. De facto, a International Agency for Research on Cancer (IARC) classifica 107 agentes como carcinogénios humanos confirmados (Grupo 1), dos quais 26 exercem os seus efeitos a nível pulmonar; destes, apenas dois não o são por via de exposição ocupacional - o carvão usado no aquecimento das casas e na cozinha e o tabagismo ativo (Yu, Tse, \& Qiu, 2012).

Estima-se que 10-25\% dos cancros do pulmão em todo o mundo ocorrem em não-fumadores, ou seja, indivíduos que fumaram menos de 100 cigarros em toda a vida. O tipo histológico mais frequente é o adenocarcinoma. A exposição ao fumo de tabaco ambiental (particularmente em mulheres) e a exposição a agentes carcinogénios no local de trabalho (particularmente em homens) são os dois mais importantes fatores de risco (Couraud et al., 2012, De Matteis et al., 2013).

O cancro do pulmão é o cancro ocupacional mais bem estudado e a exposição prévia a carcinogénios ocupacionais é um dos seus mais importantes determinantes (Yu, Tse, \& Qiu, 2012).

São numerosos os agentes em que já existe evidência epidemiológica suficiente para estabelecer nexos de causalidade inequívocos entre exposição ocupacional e cancro do pulmão. No Quadro 2 mencionam-se os principais.

Quadro 2. Causas ocupacionais de Cancro de Pulmão.

Adaptado de Cogliano (Cogliano et al., 2011) e de European Lung White Book (2013).

\begin{tabular}{|l|}
\hline \multicolumn{1}{|c|}{ Evidência Estabelecida } \\
\hline Produção de alumínio \\
\hline Arsénio e compostos inor gânicos de arsénio \\
\hline Amianto (todas as form as) \\
\hline Berílio e compostos de berilio \\
\hline Bis (clorometil) éter \\
\hline Clorometil-m etiléter \\
\hline Cádmio e compostos de cádmio \\
\hline Compostos de cró mio hexavalente \\
\hline Carvão-em issões derivadas da com bust ão doméstica \\
\hline Gaseificação de carvão \\
\hline Coque- produção \\
\hline Alcatrão de carvão -"coal tar pitch " \\
\hline Mineração de hematite \\
\hline Fundição de ferro e aço \\
\hline MopP (mecloretam ina, vincristina, procarbazina e \\
prednisona) \\
\hline Compostos de níquel \\
\hline Algumas tintas especificas \\
\hline Plutónio \\
\hline Radão-222 e seus produtos de decaimento \\
\hline Indústria de produ ção de borracha \\
\hline Sílica cristalina \\
\hline Fuligem \\
\hline Mostarda de enxofre \\
\hline Fumo de tabaco am biental \\
\hline Radiação X, radiação gama \\
\hline
\end{tabular}

\begin{tabular}{|l|}
\hline \multicolumn{1}{|c|}{ Evidência Limitada } \\
\hline Aerossóis de misturas ácid as inorgânicas fortes \\
\hline Vid ro-produção especifica \\
\hline Biomassa-emissões derivadas da combustão dom éstica \\
\hline Elét rodos de carbono- produção \\
\hline $\begin{array}{l}\text { Tolueno alfa clorinado e cloreto de benzo ilo (exposição } \\
\text { combinada) }\end{array}$ \\
\hline Cobalto metálico com carboneto de tungsténio \\
\hline Creosoto \\
\hline Emissões de veiculos diesel \\
\hline Frituras - emissões a altas tem peraturas \\
\hline Insecticidas (não arsenicais) \\
\hline Processões de impressão específicos \\
\hline 2, 3, 7, 8-tetraclorodibenzo-p-dioxina \\
\hline Fumos de soldadu ra \\
\hline
\end{tabular}


Entre as várias exposições ocupacionais mais relacionadas com cancro ocupacional, destacam-se algumas:

Amianto: é um dos riscos ocupacionais mais minuciosamente caracterizados e investigados, quer no que toca à etiologia do cancro do pulmão quer da pleura (mesotelioma pleural) (Lenters et al., 2011). Inúmeros modelos de avaliação de risco têm sido desenvolvidos na tentativa de fornecer informações confiáveis sobre os riscos de cancro de pulmão no local de trabalho. Os próprios métodos de amostragem e análise do amianto têm mudado dramaticamente ao longo dos anos.

Globalmente, a cada ano, cerca de 125 milhões de pessoas ainda estão expostas ocupacionalmente ao amianto (WHO 2006). Exposição por inalação, e em menor extensão, por ingestão, ocorre na mineração e na moagem de amianto (ou outros minerais contaminados com amianto), no fabrico ou na utilização de produtos que contenham amianto, na construção, na indústria automóvel, nas atividades de remoção de amianto (incluindo o transporte e eliminação de resíduos contendo estas fibras) (IARC, 2009).

A associação entre a exposição ao amianto e o risco de cancro de pulmão é basicamente linear, exceto em exposições muito elevadas. Muitos estudos demonstram que o risco relativo para cancro de pulmão aumenta entre $1 \%$ e $4 \%$ por fibra-ano/mL de exposição, correspondendo a uma duplicação do risco nos valores de 25-100 fibras-ano/mL. No entanto, um estudo de elevada qualidade mostrou uma duplicação do risco com valores de exposição de apenas cerca de 4 fibras-ano $/ \mathrm{mL}$. $O$ tipo histológico e a localização do cancro do pulmão não são úteis na diferenciação de outros cancros de pulmão não induzidos pelo amianto. A presença de placas pleurais, corpos de amianto ou fibras de amianto são úteis como marcadores de exposição prévia. Todos os tipos de amianto estão potencialmente associados com cancro de pulmão. Por outro lado, relativamente ao risco oncológico pulmonar, a interação entre o amianto e o tabagismo apresenta caraterísticas que o situa entre aditivo e o multiplicativo (Nielsen et al., 2014).

O mesotelioma pleural é um tumor raro que ocorre nas células mesoteliais que recobrem a membrana pleural. Os casos não associados a exposição ocupacional ou ambiental a amianto são raríssimos, com uma incidência anual estimada de 1 a 2 por milhão nos EUA (Begin et al., 1992). A sua relação causa-efeito foi inicialmente descoberta após estudos de Wagner e col. na África do Sul, em casos com exposição ambiental e ocupacional (Wagner, Sleggs,\& Marchand 1960). De uma forma geral, as populações com exposição a amianto (sobretudo crocidolite) têm um risco cerca de 5 a 20 vezes superior de desenvolvimento de mesotelioma (Begin et al., 1992). Trata-se de um tumor que ocorre mais frequentemente após a exposição a fibras anfíbolas, face ao amianto crisótilo. $O$ risco estimado de mesotelioma associado a um aumento de uma unidade (em fibras/ano) no que toca a exposição a fibras anfíbolas de amianto é considerável (IARC, 2009).

Radão - O radão-222 é um gás radioativo que ocorre naturalmente e é responsável por cerca de metade da exposição humana mundial a radiação. A exposição crónica ao radão e seus produtos de decaimento é estimada como a segunda principal causa do cancro do pulmão (após o tabaco). A radiação ionizante emitida durante o decaimento radioativo do radão e seus compostos relacionados pode induzir uma variedade de efeitos citogenéticos que resultam num aumento do risco de carcinogénese (Robertson et al., 2013).

Taxas elevadas de cancro de pulmão têm sido relatadas a partir de vários estudos de coorte e caso-controlo de mineiros expostos ao radão e seus produtos de decaimento. Estes estudos incluem principalmente mineiros de urânio, mas também da mineração de ferro, fluorite e outros metais. Fortes evidências de relações exposição-resposta foram obtidas a partir de vários desses estudos, apesar de algumas incertezas que afetam as estimativas da exposição das populações em estudo. Vários estudos caso-controlo do cancro do pulmão têm sugerido também um risco maior entre os indivíduos que vivem em habitações com valores de exposição mais elevados, havendo uma sinergia entre exposição ocupacional e ambiental (IARC, 1988).

Sílica Cristalina - Devido à extensa ocorrência natural de sílica cristalina na crosta terrestre e os amplos usos dos materiais em que é um constituinte, os trabalhadores podem ser expostos à sílica cristalina numa grande variedade de indústrias e profissões (IARC, 2009).

Berílio - É um dos metais mais leves, com um dos pontos de fusão mais elevados e com uma grande condutividade térmica, o que o tornou amplamente usado na indústria de armamento, nuclear, de telecomunicações, em ligas metálicas resistentes, em prótese dentária e na indústria aeroespacial e automóvel.

A IARC classifica o berílio como carcinogénio do grupo 1, uma vez que foram realizados vários estudos envolvendo exposições ocupacionais a este metal, nomeadamente em unidades de processamento de berílio (Sanderson, Petersen, \& Ward, 2001; Steenland \& Ward, 1991; Ward et al., 1992; IARC, 2009).

Fumo de tabaco ambiental - uma vez que em muitos países só mais recentemente foram adotadas leis de proteção antitabágica nos espaços públicos e locais de trabalho, muitos cancros pulmonares ocupacionais podem resultar especificamente da exposição a fumo de tabaco ambiental.

Revista Internacional em Língua Portuguesa, 2018, №34, Ciências da Saúde e Tecnologia, pp. 53-76 
Desde 1981, mais de 50 estudos epidemiológicos examinaram a associação entre o fumo de tabaco ambiental e cancro do pulmão, resultando na conclusão de que a exposição de não fumadores é causalmente associada com um aumento de risco de cancro do pulmão, o que levou a IARC a classificar o fumo de tabaco ambiental como carcinogénio do grupo 1 (IARC 2004). Fumos da Combustão de diesel - Vários estudos, incluindo uma revisão internacional pela IARC, demonstraram que as emissões dos veículos a diesel aumentam o risco de cancro do pulmão, com um OR de 1,8 (IC 95\%: 1,2-2,6) (Pintos et al., 2012; Benbrahim-Tallaa et al., 2012).

Em conclusão, a patologia respiratória de etiologia ocupacional representa uma vasta e heterogénea família de potenciais quadros clínicos de gravidade muito variável, mas passíveis de induzir significativa morbilidade e mortalidade nas populações afetadas.

Só o seu reconhecimento precoce e sobretudo a adoção e o investimento em medidas de Prevenção Primária (e também Secundária) poderá modificar o quadro atual que se observa a nível mundial. Tem aqui papel preponderante, na área respiratória, a atuação médica, nomeadamente da especialidade de Medicina do Trabalho, Pneumologia, Imunoalergologia, Medicina Interna (entre outras) e a atuação não médica, desenvolvida por todos os outros profissionais diferenciados e potencialmente envolvidos na abrangente e multidisciplinar área da Saúde Ocupacional.

\section{Referências}

Agostini, C., Trentin, L., Facco, M., \& Semenzato, G. (2004). New aspects of hypersensitivity pneumonitis. Curr Opin Pulm Med no. $10(5), 378-82$.

Ahasic, A. M., \& Christiani, D. C. (2011). Respiratory Disorders. In Occupational and environmental health: recognizing and preventing disease and injury, edited by Barry S. Levy. Oxford: Oxford University Press.

Akira, M. (2002). High-resolution CT in the evaluation of occupational and environmental disease. Radiol Clin North Am no. 40(1), 43-59.

American Thoracic Society. Medical Section of the American Lung Association: The diagnosis of nonmalignant diseases related to asbestos. (1986). Am Rev Respir Dis no. 134(2), 363-8.

Bakke, P. S., Baste, V. Hanoa, R. \& Gulsvik, A. (1991). Prevalence of obstructive lung disease in a general population: relation to occupational title and exposure to some airborne agents. Thorax no. 46(12), 863-70.

Bang, K. M., Weissman, D. N., Pinheiro, G. A., Antao, V. C., Wood, J. M., \& Syamlal, G. (2006). Twenty-three years of hypersensitivity pneumonitis mortality surveillance in the United States." Am J Ind Med no. 49(12), 997-1004.

Banks, D. E., \& Parker, J. E. (1998.) Occupational lung disease: an international perspective. London: Chapman \& Hall.

Barbara, C., Rodrigues, F., Dias, H., Cardoso, J., Almeida, J.,Matos, M. J., Simao, P., Santos, M., Ferreira, J. R., Gaspar, M., Gnatiuc, L., \& Burney, P. (2013). Chronic obstructive pulmonary disease prevalence in Lisbon, Portugal: the burden of obstructive lung disease study. Rev Port Pneumol no. 19(3), 96-105.

Baur, X., \& Sigsgaard, T. (2012). The new guidelines for management of work-related asthma. Eur Respir J no. 39(3), 518-9.

Baur, X., Aasen, T. B., Burge, P. S., Heederik, D., Henneberger, P. K., Maestrelli, P., Schlunssen, V., Vandenplas, O., \& Wilken, D. (2012). The management of work-related asthma guidelines: a broader perspective. Eur Respir Rev no. 21(124),125-39.

Begin, R. (1999). Asbestos-related Disorders. In Occupational lung disorders ERS monograph 11, edited by C. E. Mapp. Sheffield: European Respiratory Society Journals

Begin, R., Ostiguy, G., Fillion, R., \& Colman, N. (1991). Computed tomography scan in the early detection of silicosis." Am Rev Respir Dis no. 144(3 Pt 1), 697-705.

Begin, R., Gauthier, J. J., Desmeules, M., \& Ostiguy, G. (1992). Work-related mesothelioma in Quebec, 1967-1990. Am J Ind Med no. 22(4), 531-42.

Benbrahim-Tallaa, L., Baan, R. A., Grosse, Y., Lauby-Secretan, B., El Ghissassi, F., Bouvard, V., Guha, N., Loomis, D., \& Straif, K. (2012). Carcinogenicity of diesel-engine and gasoline-engine exhausts and some nitroarenes. Lancet Oncol no. 13(7), 663-4. 
Bernstein, L. I., Bernstein, D. I., Chan-Yeung, M., \& Malo, J. L. (2006). Definition and Classification of Asthma in the Workplace. In Asthma in the workplace : and related conditions, edited by L. I. Bernstein, M. Chan-Yeung, J. L. Malo and D. I. Bernstein, 1-8. New York: Taylor \& Francis.

Blanc, P. D., \& Toren, K. (1999). How much adult asthma can be attributed to occupational factors? Am J Med no. 107(6), 580-7. doi: S0002-9343(99)00307-1 [pii].

Brichet, A., Salez, F., Lamblin, C., \& Wallaert, B. (1999). Coal Workers Pneumoconiosis and Silicosis. In Occupational lung disorders ERS monograph 11, edited by C. E. Mapp. Sheffield: European Respiratory Society Journals.

Buist, A. S., McBurnie, M. A., Vollmer, W. M., Gillespie, S., Burney, P., Mannino, D. M., Menezes, A. M., Sullivan, S. D., Lee, T. A., Weiss, K. B., Jensen, R. L., Marks, G. B., Gulsvik, A., \& Nizankowska-Mogilnicka, E. (2007). International variation in the prevalence of COPD (the BOLD Study): a population-based prevalence study." Lancet no. 370(9589), 741-50.

Burge, P. S. 81999). Occupational Chronic Obstructive Pulmonary Disease. In Occupational lung disorders, edited by C. E. Mapp, 242-254. [Sheffield]: European Respiratory Society Journals.

Cogliano, V. J., Baan, R., Straif, K., Grosse, Y., Lauby-Secretan, B., El Ghissassi, F. Bouvard, V., Benbrahim-Tallaa, L., Guha, N., Freeman, C., Galichet, L., \& Wild, C. P. (2011). Preventable exposures associated with human cancers. J Nat/ Cancer Inst no. 103(24), 1827-39.

Cordeiro, A. J., Robalo, A. (1995). Pneumologia Fundamental. Lisboa: Fundação Calouste Gulbenkian.

Cordeiro, C. R., Jones, J. C., Alfaro, T., \& Ferreira, A. J. (2007). Bronchoalveolar lavage in occupational lung diseases. Semin Respir Crit Care Med no. 28(5), 504-13.

Cormier, Y., Belanger, J., LeBlanc, P., \& Laviolette, M. (1986). Bronchoalveolar lavage in farmers' lung disease: diagnostic and physiological significance. Br J Ind Med no. 43(6), 401-5.

Costabel, U., Bonella,, F., \& Guzman, J. (2012). Chronic hypersensitivity pneumonitis. Clin Chest Med no. 33(1), $151-63$.

Couraud, S., Zalcman, G., Milleron, B., Morin, F., \& Souquet, p. J. (2012). Lung cancer in never smokers--a review. Eur J Cancer no. 48(9), 1299-311. doi: 10.1016/j.ejca.2012.03.007

De Matteis, S., Consonni, D., Pesatori, A. C., Bergen, A. W., Bertazzi, P. A., Caporaso, N. E., Lubin, J. H., Wacholder, S., \&Landi, M. T. (2013). Are women who smoke at higher risk for lung cancer than men who smoke? Am J Epidemiol no. 177(7), 601-12.

Decreto-Lei n.o 266/2007, de 24 de Julho. Diário da República, 1.a série - N.o 141 - 24 de Julho de 2007. Ministério do Trabalho e da Solidariedade Social. 2007

Diaz-Guzman, E., Aryal, S., \& Mannino, D. M. (2012). Occupational chronic obstructive pulmonar disease: an update. Clin Chest Med no. 33(4), 625-36.

Dodson, R. F., \& Hammar, S. P. (2005). Asbestos: risk assessment, epidemiology, and health effects. Boca Raton: Taylor \& Francis.

European Lung White Book. Ed. G. J. Gibson, Loddenkemper, R., Sibille, Y., \& Lundbäck, B. : Sheffield: ERS Journals. (2013).

Fink, J. N., Ortega, H. G., Reynolds, H. Y., Cormier, Y. F., LFan, L. L., Franks, T. J., Kreiss, K., Kunkel, S., Lynch, D., Quirce, S., Rose, C., Schleimer, R. P.,, Schuyler, M. R., Selman, M., Trout, D., \& Yoshizawa, Y. (2005). Needs and opportunities for research in hypersensitivity pneumonitis. Am J Respir Crit Care Med no. 171(7), 792-8.

Fujimura, N. (2000). Pathology and pathophysiology of pneumoconiosis. Curr Opin Pulm Med no. 6(2), 140-4.

Ghio, A. J. (2012). Pneumoconiosis in the Twenty-First Century. In A clinical guide to occupational and environmental lung diseases, edited by Yuh-Chin T. Huang, Andrew J. Ghio and Lisa A. Maier, 171-187. New York: Humana Press.

GOLD, (2014). Global strategy for the diagnosis, management, and prevention of chronic obstructive pulmonary disease / Global Initiative for Chronic Obstructive Lung Disease (GOLD). Updated 2014 ed.

Greenberg, M. I., Waksman, J., \& Curtis, J. (2007). Silicosis: a review. Dis Mon no. 53(8), 394-416.

Guia de boas práticas para prevenir ou minimizar os riscos decorrentes do amianto em trabalhos que envolvam (ou possam envolver) amianto, destinado a empregadores, trabalhadores e inspectores do trabalho. Ed. Comissão Europeia, 2006.

Henneberger, P. K., Redlich, C. A., Callahan, D. B., Harber, P., Lemiere, C., Martin, J., Tarlo, S. M., Vandenplas, O., \& Toren, K. (2011). An official american thoracic society statement: work-exacerbated asthma. Am J Respir Crit Care Med no. 184(3), 368-78. 
Hirschmann, J. V., Pipavath, S. N., \& Godwin, J. D. (2009). Hypersensitivity pneumonitis: a historical, clinical, and radiologic review. Radiographics no. 29(7), 1921-38.

Ho, L., \& Kuschner, W. G. (2012). Hypersensitivity Pneumonitis. In A clinical guide to occupational and environmental lung diseases, edited by Yuh-Chin T. Huang, Andrew J. Ghio and Lisa A. Maier, 113-132. New York: Humana Press.

Huang, Yuh-Chin T., Ghio, A. J., \& Maier, L. A. (2012). A clinical guide to occupational and environmental lung diseases. New York: Humana Press.

IARC, (1988). Man-made mineral fibres and radon. IARC Monographs on the Evaluation of Carcinogenic Risks to Humans Vol. 43. Lyon, France.

IARC, (2004). Tobacco Smoke and Involuntary Smoking. IARC Monographs on the Evaluation of Carcinogenic Risks to Humans Vol. 83. Lyon, France.

IARC, (2009). A Review of Human Carcinogens: Arsenic, Metals, Fibres, and Dusts. IARC Monographs on the Evaluation of Carcinogenic Risks to Humans Vol 100 C. Lyon, France.

ILO; (2011). Guidelines for the use of the ILO International Classification of Radiographs of Pneumoconioses. Edited by ILO. Geneva: ILO.

ILO, (2013). The Prevention of Occupational Diseases. edited by International Labour organization. Geneva.

Johansson, S. G., Hourihane, J. O., Bousquet, J., Bruijnzeel-Koomen, C., Dreborg, S., Haahtela, T., Kowalski, M. L., Mygind, N., Ring, J., van Cauwenberge, P., van Hage-Hamsten, M., \& Wuthrich, B. 82001). A revised nomenclature for allergy. An EAACI position statement from the EAACl nomenclature task force. Allergy no. 56(9), 813-24.

Korn, R. J., Dockery, W. D., Speizer, E. f., Ware, J. W., \& Ferris, B. G., (1987). Occupational exposures and chronic respiratory symptoms. A population-based study. Am Rev Respir Dis no. 136(2), 298-304.

Lacasse, Y., Girard, M., \& Cormier, Y. (2012). Recent advances in hypersensitivity pneumonitis. Chest no. 142(1)208-17.

Lemiere, C., Boulet, L. P., Chaboillez, S., Forget, A., Chiry, S., Villeneuve, H., Prince, P., Maghni, K., Kennedy, W. A, \& Blais, L. (2013). Work-exacerbated asthma and occupational asthma: do they really differ? J Allergy Clin Immunol no. 131(3), 704-10.

Lenters, V., Vermeulen, R., Dogger, S., Stayner, L., Portengen, L., Burdorf, A., \& Heederik, D. (2011). A meta-analysis of asbestos and lung cancer: is better quality exposure assessment associated with steeper slopes of the exposure-response relationships?" Environ Health Perspect no. 119(11), 1547-55. doi: 10.1289/ehp.1002879.

Leung, C. C., Yu, I. T., \& Chen, W. (2012). Silicosis. Lancet no. 379(9830),2008-18.

Lopez, A. D., Shibuya, K., Rao, C., Mathers, C. D., Hansell, A. L., Held, L. S., Schmid, V., \& Buist, S. (2006). Chronic obstructive pulmonary disease: current burden and future projections. Eur Respir J no. 27(2)397-412.

Malo, J. L., \& Vandenplas, O. (2011). Definitions and classification of work-related asthma. Immunol Allergy Clin North Am no. 31(4)645-62, v.

Mapp, C. E., \& Boschetto, P. (2003). Occupational asthma. Edited by K. Fan Chung and L. M. Fabbri, Asthma. Sheffield: European Respiratory Society Journals.

Mathers, C. D., \& Loncar, D. (2006). Projections of global mortality and burden of disease from 2002 to 2030. PLoS Med no. 3(11), e442.

Morais, A., Winck, J. C., Delgado, L., Palmares, M. C., Fonseca, J., Moura e Sa, J., \& JMarques, J. A. (2004). Suberosis and bird fancier's disease: a comparative study of radiological, functional and bronchoalveolar lavage profiles. J Investig Allergol Clin Immunol no. 14(1), 26-33.

Nicholson, P. J., Cullinan, P., \& Burge, S. (2012). Concise guidance: diagnosis, management and prevention of occupational asthma. Clin Med no. 12(2), 156-9.

Nielsen, L. S., Baelum, J., Rasmussen, J., Dahl, S., Olsen, K. E., Albin, M., Hansen, N. C., \& Sherson, D. (2014). Occupational asbestos exposure and lung cancer--a systematic review of the literature." Arch Environ Occup Health no. 69(4), 191-206.

Pintos, J., Parent, M. E.,Richardson, L., \& Siemiatycki, J. (2012). Occupational exposure to diesel engine emissions and risk of lung cancer: evidence from two case-control studies in Montreal, Canada. Occup Environ Med no. 69(11), 787-92. 
Pipavath, S. N., Godwin, J. D.,\& Kanne, J. P. (2010). Occupational lung disease: a radiologic review. Semin Roentgenol no. 45(1), 43-52.

Ribeiro, M., \& Tarlo, S. M. (2012). Environmental and Occupational Causes of Asthma. In A clinical guide to occupational and environmental lung diseases, edited by Yuh-Chin T. Huang, Andrew J. Ghio and Lisa A. Maier, 93-112. New York: Humana Press.

Robertson, A., Allen, J., Laney, R., \& Curnow, A. (2013). The cellular and molecular carcinogenic effects of radon exposure: a review. Int J Mol Sci no. 14(7), 14024-63.

Salvaggio, J. E., Vockroth, A. E., \& Lopez, M. (1998). Hypersensitivity Pneumonitis. In Occupational lung disease: an international perspective, edited by D. E. Banks and J. E. Parker, 435-451. London: Chapman \& Hall.

Salvi, S. (2014). Tobacco smoking and environmental risk factors for chronic obstructive pulmonar disease. Clin Chest Med no. 35(1), 17-27.

Sanderson, W. T., Petersen, M. R., \& Ward, E. M. (2001). Estimating historical exposures of workers in a beryllium manufacturing plant. Am J Ind Med no. 39(2), 145-57.

Santos, C., Norte, A., Fradinho, F., Catarino, A., Ferreira, A. J., Loureiro, M., \& Baganha, M. F. (2010). Silicosis - brief review and experience of a pulmonology ward. Rev Port Pneumol no. 16(1), 99-115.

Savranlar, A., Altin, R., Mahmutyazicioglu, K., Ozdemir, H., Kart, L., Ozer, T., \& Gundogdu, S. (2004). Comparison of chest radiography and high-resolution computed tomography findings in early and low-grade coal worker's pneumoconiosis. Eur $J$ Radiol no. 51(2), 175-80.

Steenland, K., \& Ward, E. (1991). Lung cancer incidence among patients with beryllium disease: a cohort mortality study. J Natl Cancer Inst no. 83(19), 1380-5.

Sunyer, J., Zock, J. P., Kromhout, H., Garcia-Esteban, R, Radon, K., Jarvis, D., Toren, K., Kunzli, N., Norback, D., d’Errico, A., Urrutia, I., Payo, F., Olivieri, M., Villani, S., Van Sprundel, M., Anto, J. M., \& Kogevinas, M. (2005). Lung function decline, chronic bronchitis, and occupational exposures in young adults. Am J Respir Crit Care Med no. 172(9), 1139-45.

Szema, A. M. (2012). Work-exacerbated asthma. Clin Chest Med no. 33(4), 617-24.

Tan, J., \& Bernstein, J. A. (2014). Occupational asthma: an overview. Curr Allergy Asthma Rep no. 14(5), 431. doi: 10.1007/s11882-014-0431-y.

Tarlo, S. M., Balmes, J., Balkissoon, R., Beach, J., Beckett, W., Bernstein, D., Blanc, P. D., Brooks, S. M., Cowl, C. T., Daroowalla, F., Harber, P., Lemiere, C., Liss, G. M., Pacheco, K. A., Redlich, C. A., Rowe, B., \& Heitzer, J. (2008). Diagnosis and management of work-related asthma: American College Of Chest Physicians Consensus Statement. Chest no. 134(3 Suppl), 1S-41S.

Thaon, I., Reboux, G., Moulonguet, S., \& Dalphin, J. C. (2006). [Occupational hypersensitivity pneumonitis]. Rev Mal Respir no. 23(6), 705-25.

Thomeer, M. J., Costabel, U., Rizzato, G., Poletti, V., \& Demedts, M. (2001). Comparison of registries of interstitial lung diseases in three European countries. Eur Respir J Suppl no. 32:114s-118s.

Tossavainen, A. (1997). Asbestos, asbestosis, and cancer: the Helsinki criteria for diagnosis and attribution. Scand J Work Environ Health no. 23(4), 311-6. doi: 226 [pii].

UONIE/ACSS. (2008). Guia para procedimentos de inventariação de materiais com amianto e acções de controlo em unidades de saúde - G 03/2008 edited by UONIE/ACSS. Lisboa.

Vandenplas, O., and Malo, J. L. (2003). Definitions and types of work-related asthma: a nosological approach. Eur Respir J no. 21(4), 706-12.

Wagner, J. C., Sleggs, C. A., \& Marchand, P. (1960). Diffuse pleural mesothelioma and asbestos exposure in the North Western Cape Province. Br J Ind Med no. 17:260-71.

Ward, E., Okun, A., Ruder, A., Fingerhut, M., \& Steenland, K. (1992). A mortality study of workers at seven beryllium processing plants. Am J Ind Med no. 22(6), 885-904.

WHO, (2006). Elimination of Asbestos Related Diseases. World Health Organization. 
Winck, J. C., Delgado, L., Murta, R., Lopez, M., \& Marques, J. A. (2004). Antigen characterization of major cork moulds in Suberosis (cork worker's pneumonitis) by immunoblotting. Allergy no. 59(7), 739-45.

Yu, I. T. S., Tse, L., \& Qiu, H. (2012). Occupational Lung Cancer. In A clinical guide to occupational and environmental lung diseases, edited by Yuh-Chin T. Huang, Andrew J. Ghio and Lisa A. Maier, 251-264. New York: Humana Press.

Zacharisen, M. C., \& Fink, J. N. (2011). Hypersensitivity pneumonitis and related conditions in the work environment. Immunol Allergy Clin North Am no. 31(4), 769-86, vii.

Zock, J. P., Sunyer, J., Kogevinas, M., Kromhout, H., Burney, P., \& Anto, J. M. (2001). Occupation, chronic bronchitis, and lung function in young adults. An international study. Am J Respir Crit Care Med no. 163(7), 1572-7.

\section{Sobre os Autores}

António Jorge Correia de Gouveia Ferreira (PhD) é professor Auxiliar da Faculdade de Medicina da Universidade de Coimbra. É médico especialista em Pneumologia e em Medicina do Trabalho. É assistente hospitalar graduado do Serviço de Pneumologia A do Centro Hospitalar e Universitário de Coimbra/Hospitais da Universidade de Coimbra, onde é responsável desde 2004 pela Consulta de Patologia Profissional Respiratória. É pós-graduado em Medicina do Trabalho, Mestre em Saúde Ocupacional e Doutorado em Ciências da Saúde, na área de Medicina Preventiva e Comunitária, pela Universidade de Coimbra. É docente nas áreas de Medicina Preventiva, Saúde Comunitária, Saúde Ocupacional, Saúde Pública e Pneumologia. É coordenador do Mestrado em Saúde Ocupacional e do Curso de Pós-Graduação em Medicina do Trabalho da Faculdade de Medicina da Universidade de Coimbra. Desempenha funções de vice-presidente da Associação de Estudos Respiratórios (AER). É membro eleito do Conselho Pedagógico desta Faculdade, onde também faz parte da equipa do Gabinete de Estudos Avançados e do Instituto de Higiene e Medicina Social. 